$17^{\text {th }}$ International Congress of Metrology, 09003 (2015)

DOI: $10.1051 /$ metrology / 201509003

(C) Owned by the authors, published by EDP Sciences, 2015

\title{
Metrology for radiotherapy using complex radiation fields - HLT09 EMRP Project
}

\author{
Jean-Marc Bordy ${ }^{1}$,, Claus Andersen ${ }^{2}$, Ulrike Ankerhold ${ }^{3}$, Veronique Dedieu ${ }^{4}$, Frank Delaunay ${ }^{1}$, Jacco De Pooter ${ }^{5}$, Jarolav \\ Compell $^{6}$, Simon Duane ${ }^{7}$, Ralf-Peter Kapsch ${ }^{3}$, Antti Kosunen ${ }^{8}$, Gabor Machula ${ }^{9}$, Marco Marinelli ${ }^{12}$, Hugo Palmans ${ }^{7}$, Maria \\ Pimpinella ${ }^{10}$, Massimo Pinto ${ }^{10}$, Gianluca Verona Rinati ${ }^{12}$ and Jaroslav Solc ${ }^{11}$
}

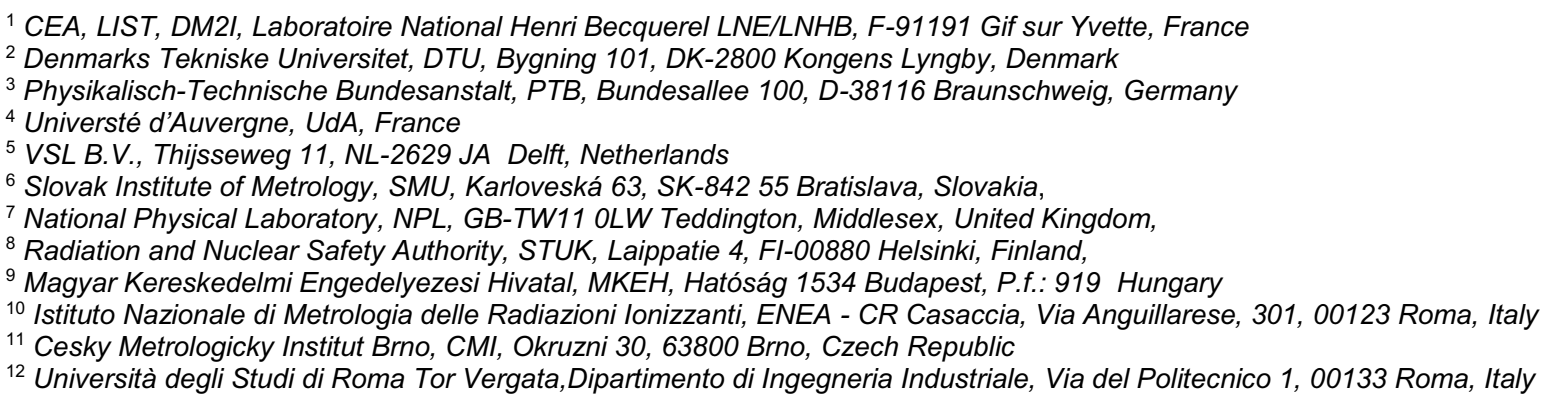

Résumé. Cette publication traite des avancées effectuées pendant le cours du projet HLT09 subventionné par EMRP (European Metrology Research Program). Ce projet avait pour objectif global d'améliorer la traçabilité, à des références nationales, de la dose absorbée délivrée à la tumeur dans la cadre d'un traitement par radiothérapie. Les recherches couvrent l'établissement de références absolues en termes de dose absorbée dans l'eau pour les photons d'énergie moyenne, la mise en œuvre du concept de produit dose surface dans la cadre de l'utilisation des faisceaux de photons de haute énergie, la caractérisation des faisceaux produit par de nouveaux générateurs de rayons X pour la thérapie de contact incluant les étalon primaire et de transfert, l'étude des caractéristiques des détecteurs ponctuels, $2 \mathrm{D}$ et $3 \mathrm{D}$ en tant qu'étalon de transfert et moyen de contrôle des plans de traitement.

\section{Background}

Advances in technology have enabled the introduction of complex forms of radiotherapy for the treatment of cancer, in which the dose is delivered in ways that are far removed from established reference dosimetry conditions. While treated volumes can now conform closely to the defined target, so reducing damage to surrounding normal tissue, the accuracy with which the dose is delivered may fall short of the requirements given by ICRU Report 24 and 42 (maximum error of $5 \%$ on the dose in the target volume).

Too low a dose in the target volume increases the chance of treatment failure through recurrence of the cancer while too high a dose can result in higher incidence of severe side effects. Lack of traceability to established reference dosimetry and primary standards makes it more difficult to meet the requirement of ICRU Reports. The main reasons for this are the lack of primary standards of absorbed dose to water, the gap between the standard reference and clinical conditions laying emphasis on the need for quality controls and in vivo dosimetry methods which should be easier to handle and more accurate.

The project lasted three years until June 2015; it gathered 12 partners through Europe: 10 national metrological institutes and two universities (see the list of authors). Many clinical centres were involved giving access to their irradiation facilities specially e.g. for hadron beams such as Clatterbridge Cancer Centre UK (Static $60 \mathrm{MeV}$ Proton Beam), Kern-Fysisch Versneller Instituut Groningen, The Netherlands (Scanned $190 \mathrm{MeV}$ Proton
Beam) and Heidelberg Ion Therapy Centre, Germany (Scanned 430/u Carbon Ion Beam).

\section{Scientific and technical objectives}

In order to improve the situation it is necessary to improve all steps of the metrological chain from the primary standards through to verification of the dose in and around the tumour, namely: developing new primary standards of absorbed dose to water; studying new detectors and improving the knowledge of the characteristics of existing detectors useable for quality control and in vivo dosimetry; and publishing guide lines of good practice on their use. To achieve these goals, the first stage is to develop new primary standards where a lack of such reference instruments complying with international protocols is identified i.e. for new electronic brachytherapy sources, for small size high-energy X-ray beams and for hadrons especially for scanned beams. For high-energy X-rays in radiotherapy, the possibility to measure a new integral quantity similar to dose area product (DAP) is evaluated. Whatever the radiation beams, dosimetric characteristics i.e. directional and energy response, perturbation factors, linearity, homogeneity and spatial resolution depending on the transfer dosimeters and their calibration protocols are also studied to finally propose robust protocol for in vivo dosimetry and quality control and to evaluate the possibility for assessing absorbed dose to water at a point in small beams. The dosimeters that are studied (calorimeters, ion chambers, silicon diodes, CVD diamond, ESR/Alanine, radio-chromic and storage film,

\footnotetext{
a Email de correspondance de l'auteur : jean-marc.bordy@cea.fr
} 
EPID and 3D gel) have been chosen in order to address the need for point, 2D and 3D measurements.

\section{Main results}

A new concept of mixed water/graphite calorimeter with sensitive elements immersed in water at $2 \mathrm{~cm}$ depth has been developed by ENEA to assess absorbed dose to water at $2 \mathrm{~cm}$ depth (figure 1). As the reference medium for radiotherapy is water, MC calculations have been made for an accurate evaluation of factors to convert absorbed dose to graphite into absorbed dose to water. In addition to the ENEA primary standard, there is now four primary references in Europe based on different measuring device designs (LNE-LNHB, PTB, VSL) allowing their comparison. The first results of this comparison showed deviation smaller than $+/-1 \%$. Another standard based on extrapolation chamber (figure 2) was greatly improved at MKEH and shall lead to the establishment of a fifth primary standard for medium energy X-ray in Europe.

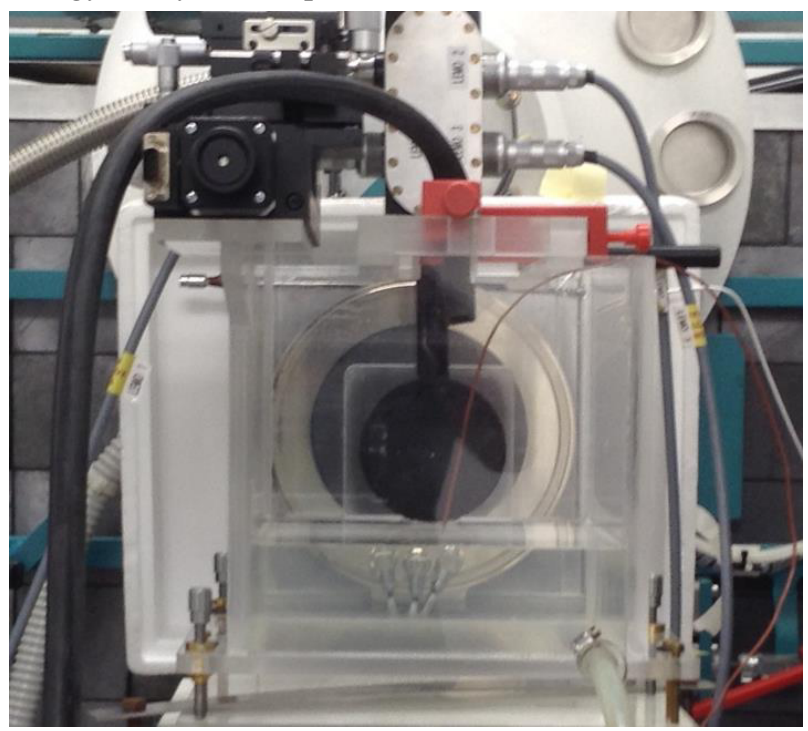

Figure 1: The mixed graphite-water ENEA calorimeter.

The LNE LNHB specially designed large area graphite calorimeter (figure 3 ) able to be used in small radiation fields (smaller than $2 \times 2 \mathrm{~cm}^{2}$ ), similar to those used in SRS and SRT (Stereotactic Radio Surgery and Radio Therapy) and IMRT (Imaging Modulated Radio Therapy), is operational. The sensitive area of such a calorimeter is larger than the field dimensions so that it measures the energy deposited by the whole radiation beam and the measured quantity is no longer a dose defined at a point, but a surface quantity similar to a dose area product (DAP). This device allows, for the first time for used for radio diagnostic, to overcome the lack of electronic equilibrium in small fields which makes the

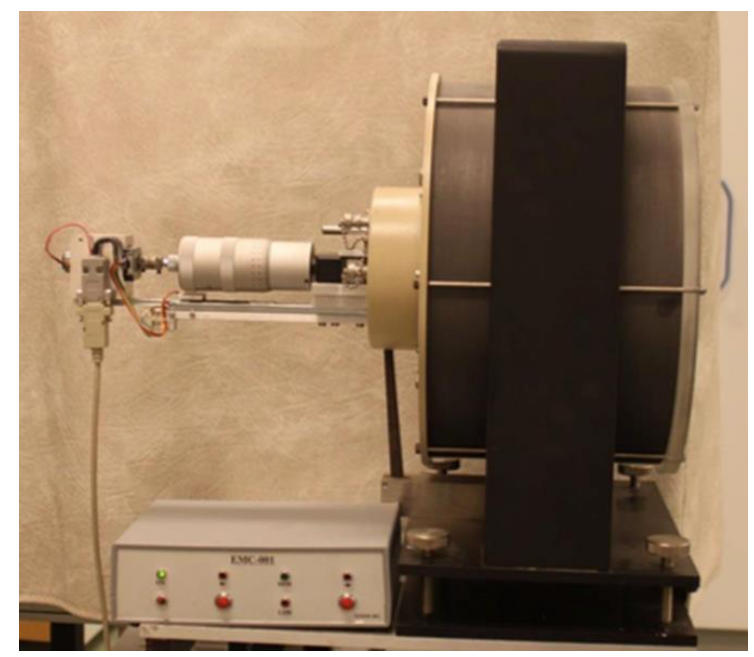

Figure 2: The MKEH extrapolation chamber

radiotherapy, the use of the DAP concept, previously point dose quantity unsuitable. Measurements in small fields down to $0.75 \mathrm{~cm}$ of diameter with specially designed collimators shows the feasibility of DAP measurements for high energy photons. Transfer standards based on homemade and commercially available ionisation chambers as well as DAP ratio between $20 \mathrm{~cm}$ and $10 \mathrm{~cm}$ (DAPR) as a quality index were tested. It turns that correction factors are needed if the transfer standard and the calorimeter have not the same sensitive surface and that the calibration coefficient of homemade secondary standard vary of about $1 \%$ depending on the beam diameter up to $2 \mathrm{~cm}$. The results are very encouraging and further studies are needed to check the behaviour of the DAP quantity in clinical situations where the radiation field is delimited by a multi-leaves collimator rather than specially designed collimators. As the DAP quantity is not implemented in treatment planning system, provided by the LINAC manufacturer, promising results have been found to convert the DAP into evaluated point absorbed dose using 2D film dosemeters.

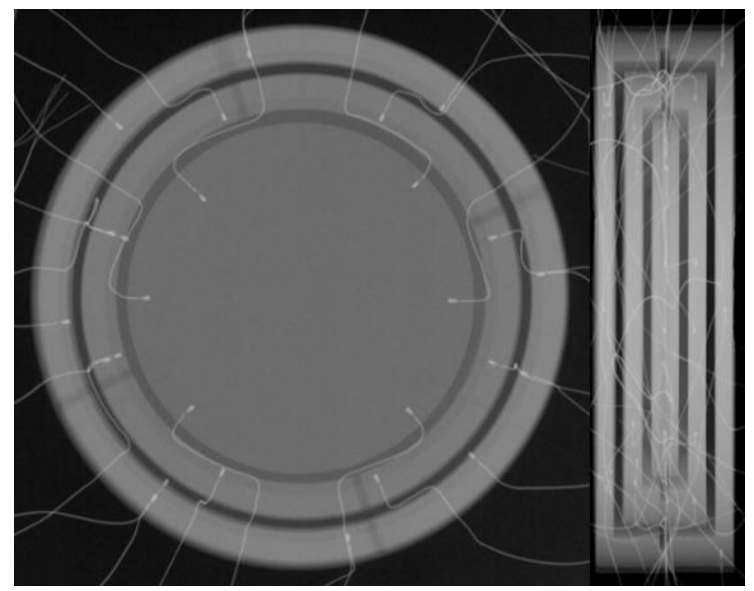

Figure 3: Top and side radiography of the LNE-LNHB graphite calorimeter detector.

PTB studied the use of point like detectors to overcome the lack of primary standards for very small radiation fields. The spatial response of commercially available ionisation chambers and diodes was studied in high energy photon and electron beams by lead, Monte-Carlo 
simulation confirmed these results but the lack of reproducibility from one chamber to another one of the same type does not allow providing generic correction factors.

For hadron therapy, a new smaller water calorimeter was built for absorbed dose measurements in a $190 \mathrm{MeV}$ proton beam and a $430 \mathrm{MeV} / \mathrm{u}$ carbon ion beam. The study of the chemical heat defect of water allows deriving correction factors. The recombination corrections for ion chambers under partial and time dependent irradiation conditions has been derived from Monte Carlo simulated dose distributions for proton beams. The fluence correction for materials such as plastic WT1, PW, PWDT, A150, polystyrene and polyethylene, and for detectors like alanine and radiochromic film has been evaluated. The energy dependence of alanine in scanned proton beams has been experimentally determined.

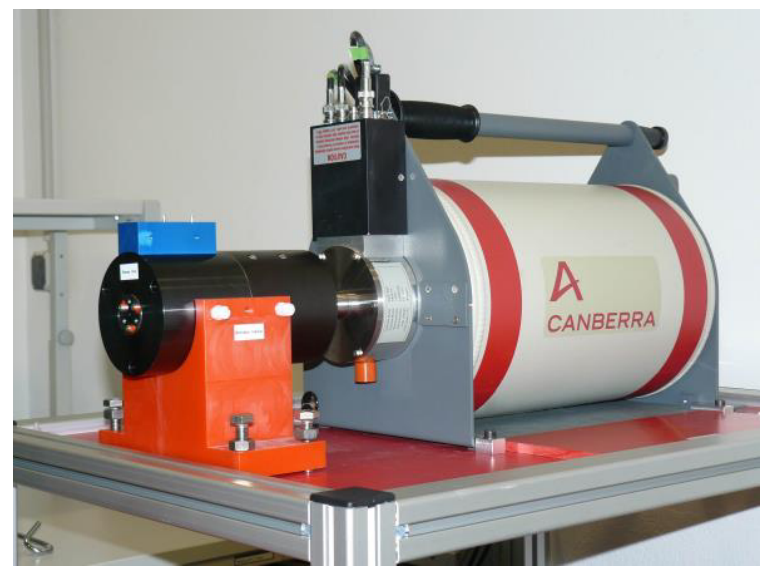

Figure 4: PTB Ge spectrometer assembly

New low energy micro x-rays generators are now available for improving the efficiency of low energy brachytherapy treatments and avoiding the use of radioactive isotopes. There currently is a lack of metrological chain from the primary standard to the end user's calibration and treatment verification to give recommendations on a calibration chain for this new treatment modality. CMI and PTB worked to overcome these lacks. The first task, before establishing a primary standard in terms of absorbed dose to water, is to characterise the sources. Radiations fields produced by INTRABEAM and AXXENT have been characterised by means of spectrometry (figure 4) and Monte Carlo calculation. Correction of pile up for continuous spectra spectrometry is done successfully. The Monte Carlo models of the micro $\mathrm{x}$-rays generator produced by Carl Zeiss (the Intrabeam) and the Xoft Axxent tube have been constructed by CMI. 3D dose distribution in water is investigated using X-ray storage foils and a plastic scintillator device developed at PTB. X-ray storage films have been characterised in term of absorbed dose to water for energy range $8-50 \mathrm{keV}$, as well as radiochromic gel in terms of energy dependence and spatial resolution. The determination of interaction coefficient for phantom material was made to finalise the reconstruction of the extrapolation chamber. For High Dose Rate sources, the influence of the dose distribution of the two typical applicators used in clinics has been experimentally investigated; dose measurements were performed with standard uncertainty less than $3 \%$.

The PTW firm makes new synthetic diamond detectors developed by ENEA and Tor Vergata Rome University commercially available as a transfer dosemeter (figure 5). $\mathrm{K}_{\mathrm{Q}, \mathrm{Q} 0}$ values were measured for electron energies in the range 6 and $15 \mathrm{MeV}$. Former attempts to use synthetic or natural based diamond detector as secondary standard failed because of the lack of reproducibility. Real time in vivo dosimetry is one of the key issues of radiotherapy because it allows verifications during the treatment and therefore avoids errors. The Tor Vergata diamond detectors are optimised in a new configuration suitable for in vivo measurements.

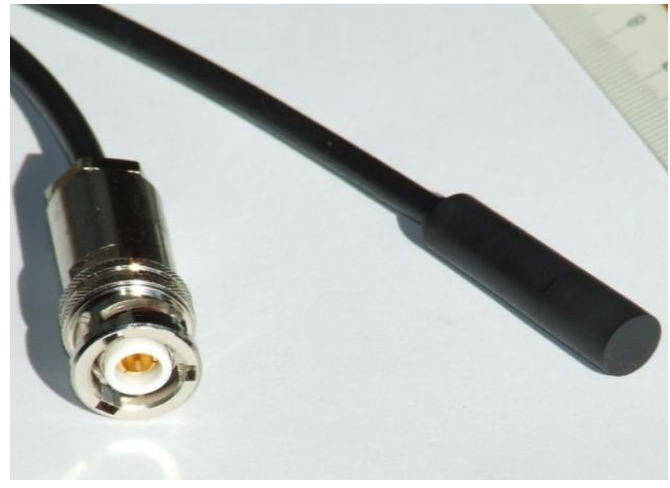

Figure 5: prototype of the Tor vergata/ENEA diamond based dosemeter.

In addition to the work on point detectors, the goal of the research program is to characterise 2D (e.g. EBT3 gafchromic film, flexible storage phosphor plates and EPID) and 3D (gel) detectors to be used for verification of complex dose distributions in photon and electron beams. The reading protocol for EBT3 gafchromic film has been optimised and the first results of STUK show that $1 \%$ accuracy in relative dose can be achieved. Spatial resolution and uncertainty of dose measurement with radiochromic film have been determined. Test for depth dose and profile measurement showed a good agreement with point detectors. One of the most promising dosimetry technic is the $3 \mathrm{D}$ dosimetry gel because it gives access in one measurement to the $3 \mathrm{D}$ distribution of the dose in a volume. Fricke gels suffer from a diffusion of the species produced by the interaction of the radiations after irradiation. The composition a special Fricke gel has been optimised to insure the require sensitivity and stability over time at least 5 hours after irradiation. A special manufacturing protocol has been optimised to insure the reproducibility of the gel. A first evaluation of the energy response exhibits a variation of $20 \%$ over the energy range few $\mathrm{kV}-20 \mathrm{MV}$. The reading method is based on MRI the reading protocol has been adapted to the new Fricke gel and the implementation of the software for the automatic analysis of the MRI image to calculate $3 \mathrm{D}$ dose distribution from the raw reading is made. This work is done in collaboration with the Université d'Auvergne (UdA) and LNE-LNHB. A new dosimetry service will be opened by UdA within the next months, the components of the gel would be sent to the medical physicist by UdA to manufacture the gel and fill in a phantom, then the phantom would be irradiated and 
read at hospital, then the raw results in DICOM format would be sent to the remote calculation centre at $\mathrm{UdA}$ which send back the $3 \mathrm{D}$ dose distribution to the treatment centres.

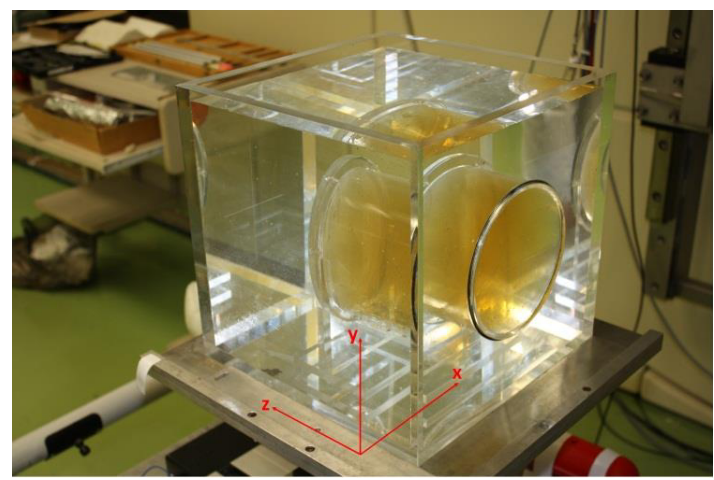

Figure 6: View of gel cylinder irradiated in a $30 \times 30 \times 30 \mathrm{~cm}^{3}$ water phantom in front of a $6 \mathrm{MV}$ LINAC photon beam.

EPID are between 2D and 3D technics because the measurement itself gives 2D information but multiple angle acquisitions and mathematical reconstruction allow the building of 3D models of the dose distributions inside the patient. As the measurement is made during the treatment, the result is a "true" dose distribution which can be used to validate the TPS calculations. NPL did the first step toward this goal that is to say to study the characteristic of the detector, EPID characteristics in terms of angular and energy responses have been measured and validated against Monte Carlo calculation, with some dependency on the (imprecisely known) detector material composition.

Using the detectors mentioned above, the aim is to develop, validate, compare and characterise (in terms of uncertainty budget) measurement methods for the 2D/3D verification of the treatment planning system. These methods can be based on one dosimeter or the association of several point, 2D, and 3D dosimeters. The validation is done in an anthropomorphic or semi anatomical phantom (to be as close as possible to the clinical conditions) on the basis of the comparison of the results of the measurement methods and TPS calculations. An anatomical women torso water phantom and 2 semianatomical phantoms - one for mastectomy and another for "head and neck" - were built by STUK and a special phantom with different tumour size for testing the ME40 organic plastic scintillator system built by DTU (figure 7). These phantoms correspond to suitable cases for investigation to be chosen among those for which the TPS calculations and the measurements have well known difficulties i.e. where high gradient doses are encountered, such as in dynamic rotational treatment modalities, and in the presence of heterogeneities and organ at risk in the vicinity of the tumour. Such phantoms are intended to be scanned for further TPS calculations and detectors are positioned within it during the simulated treatment for real time or delayed measurements of doses. A pilot study of the external quality control for IMRT (Intensity Modulated Radio Therapy) has been made with alanine put in the CIRS thorax phantom (figure 7). This study exhibits a difference of $1.6 \%$ between alanine at TPS doses while the standard uncertainty on alanine dosemeter was $1.5 \%$. For organic scintillator in the especially designed DTU phantom the reported deviations were of about $1 \%$ while the standard uncertainty on the scintillator measurement was of about $1 \%$. A series of recommendations or guidelines will be written and disseminated to the European radiotherapy community.

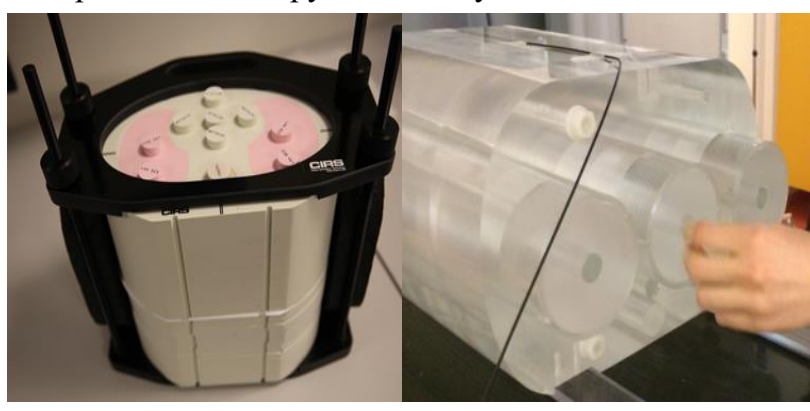

Figure 7: CIRS (left) and DTU phantoms.

\section{Conclusions}

The results of this project will enable international codes of practice for dosimetry in radiotherapy to be updated, producing good practice guidelines for quality control and in vivo dosimetry that will be distributed to the radiotherapy community. By the end of the project, the use of absorbed dose to water and its dissemination will be standardised for all types of radiation. The medical physicists will have more reliable and convenient tools available for quality control and in vivo dosimetry allowing validation of treatment plans. The consequent benefit to cancer patients of this improved dosimetry will come from better treatment outcomes.

So far, the results of the project have input into several of the American Association of Physicists in Medicine brachytherapy working groups (e.g. TG186, TG167 and TG 236) which would lead to a revision of standards. The first step to achieve this goal is to introduce the findings in an IAEA report (more international visible). Currently the German DIN standards dealing with brachytherapy are in preparation/maintenance. Contacts have also been made with the International Atomic Energy Agency (IAEA) to disseminate the project results, with a goal of updating the dosimetry protocol 398.

A workshop was organized jointly with the French medical physic society (SFPM) in June 2015 in Lille (France) to disseminate the results of this joint research program toward the stakeholders.

More information and references are available on the project website, http://radiotherapy-emrp.eu

Acknowledgement: This work was supported by EMRP (European Metrology Research Programme), Programme of EURAMET. The EMRP is jointly funded by the EMRP participating countries within EURAMET and the European Union. 